\title{
The evolutionary origin of diversity in Chagas disease vectors
}

Silvia A. Justi, Department of Biology, College of Arts and Sciences, University of Vermont, USA.silvinhajusti@gmail.com

Cleber Galvão*, Laboratório Nacional e Internacional de Referência em Taxonomia de Triatomíneos, Instituto Oswaldo Cruz, FIOCRUZ, Rio de Janeiro, Brazil. *Correspondence to clebergalvao@gmail.com

Keywords: Triatominae, diversification, phylogeny, taxonomy

\begin{abstract}
Chagas disease is amongst the ten most important neglected tropical diseases but knowledge on the diversification of its vectors, Triatominae (Hemiptera: Reduviidae), is very scarce. Most Triatominae species occur in the Americas, and are all considered potential vectors. Despite its amazing ecological vignette, there are remarkably few evolutionary studies of the whole subfamily, and only one genome sequence has been published. The young age of the subfamily, coupled with the high number of independent lineages are intriguing, yet the lack of genome wide data makes it a challenge to infer the phylogenetic relationships within Triatominae. Here we synthesize what is known and
\end{abstract}


suggest the next steps towards a better understanding of how this important group of disease vectors came to be. 


\section{Triatominae: The vectors of Chagas disease}

Chagas disease is amongst the ten most important neglected tropical diseases, being considered the most impairing protozoan parasitic infection in Latin America [1].

Transmission of Chagas disease relies on a single subfamily of insects with the ability to transmit a complex group of protozoan parasites. All vector taxa (see glossary) belonging to the Triatominae (Hemiptera:Reduviidae) subfamily are considered potentially capable of transmitting [2] all six described lineages of Trypanosoma cruzi (Kinetoplastea: Trypanosomatida) [3], even though not all of them have been found to be infected with the parasite[4]

The subfamily Triatominae is currently composed of 151 described species (149 extant and 2 fossil), assigned to 5 tribes (Table 1) [5]. The type species, Triatoma rubrofasciata, described in 1773, is the only one found in both the New and Old World. There has been only one comprehensive revision on the biology, ecology and taxonomy of the subfamily [6]; there are no published comprehensive cladistic analyses, based on morphological characters, and very few molecular phylogenetic studies target the whole group (Table S1).

Only recently, the generation of broader data on the diversification processes of Chagas disease vector allowed to better understand of Triatominae phylogeny, which is far from being deeply studied. Here, we review the current knowledge on the phylogeny of Triatominae and its major groups. 


\section{Limitations of Triatominae Phylogenetic studies}

The very first phylogenetic study of Triatominae, published in 1998 [7], investigated the relationship between eight species of Triatoma, based on three mitochondrial markers (12S, $16 \mathrm{~S}$ and cytochrome oxidase c subunit I). Since then, not much has changed concerning the number and type of molecular markers used to reconstruct Triatominae phylogeny (Table S1). Ideally, conservation of the molecular marker used in phylogenetic studies should be directly proportional to the age of the relationships of interest, meaning, the older the relationships the more conserved the markers used should be.

New markers include multi-copy nuclear ribosomal markers and a few additional nonrecombining mitochondrial markers. Only one entire mitochondrial genome has been published, that of Triatoma dimidiata [8]. This contrasts very profoundly with the published phylogenetic studies of other disease vectors, such as Aedes and Anopheles mosquitoes, which include one or more studies using a genome wide approach (e.g. $[9,10])$.

The use of such a restricted number of molecular markers is most likely due to the lack of available genetic resources, including DNA sequences and/or genomic data from closely related organisms. For instance, only six Heteroptera genome assemblies are available from GenBank (www.ncbi.nlm.nih.gov/genome) and, despite the high number of sialotranscriptomes available, the diversity of taxa from which they were obtained is still very low. The paucity of data is also observable on the database created to host disease vector-omics data, VectorBase (https://www.vectorbase.org/), which contains only one 
Triatominae genome (Rhodnius prolixus [11]) and one transcriptome (Triatoma infestans, not linked to a full publication yet), but over 20 mosquito and 6 fly genomes.

The lack of genetic resources makes it difficult to identify suitable single copy nuclear genes. Such genes are important to assure that the regions being compared are not only homologous, but also orthologous (homologous by descent, not by duplication - Figure 1).

In addition to the lack of genetic resources, a close look at published phylogenetic relationships and studies of diversification processes of Triatominae species makes it very clear that, over the years, researchers focused on species of epidemiological importance rather than attempting to understand the evolution of the whole subfamily [12]. This understandable interest in identifying the epidemiologically relevant lineages restricts our ability to understand the underling processes leading to that diversity.

Understanding the evolutionary relationships of such a diverse group of disease vectors is extremely important to generate information that can be used for vector control. With lineages adequately described and their ecological and epidemiological characteristics well studied, our increased understanding of processes contributing to diversification and domestication will facilitate better designed control strategies.

\section{Triatominae monophyly $v s$. paraphyly}

Early studies defined Triatominae as "hematophagous Reduviidae", with a starting null hypothesis that the group was monophyletic based on ecology, biology and morphology characteristics related to hematophagy [6]. 
The vast range of habitats where the different Triatominae species live and morphological similarities to other Reduviidae (specially similarities related to buccal morphology) led to competing hypotheses: that Triatominae would be either paraphyletic or polyphyletic [13-16]. Moreover, additional to hematophagy, members Triatominae have been documented to exhibit predacious behavior, [17-22] which contributes to increasing the doubts about the subfamily monophyly, defined mainly by feeding habits. Indeed, if the hematophagous feeding habit independently appeared multiple times in the subfamily, Triatominae should be considered polyphyletic (Figure 2).

The first study to show evidence of the polyphyly of Triatominae was published in 2005 and included a test of the sister status of the Triatomini and Rhodniini tribes (Figure 3, Key Figure). The study also included several Reduviidae, and using a single molecular marker, recovered Triatominae as polyphyletic [23].

Despite the conflicting evidence concerning the monophyletic status of Triatominae, until the late 2000s there were no published studies with significant Reduviidae sampling to make statistically supported inferences on the matter. The first, and to date the only, cladistics analyses, based on morphology, of the Reduviidae (including 21 of the 25 subfamilies), included a few Triatominae species, and supported the hypothesis of the subfamily to be monophyletic [24]. The sampling, however, was not designed to investigate this particular question (did not include all the Triatominae tribes, or the most closely related Reduviidae), leaving it thus, somewhat ambiguous, although the a comprehensive molecular phylogeny of Reduviidae resulted, again, in recovering the Triatominae as monophyletic [25]. 
As described for other taxa, Triatominae sampling greatly influences phylogenetic reconstruction results [26,27]; gene trees and species trees may have conflicting topologies [28]. More recently, in a 2012 study including both the widest diversity of Reduviidae and several molecular markers, Hwang and Weirauch [29] reported a phylogeny in which they observed the genera Opisthacidius and Zelurus to be closely related to Triatominae, recovered as paraphyletic. However, the gathering of this diverse sampling with the most diverse Triatominae sampling in a phylogenetic study [30] revealed a monophyletic Triatominae clade [5].

As shown above, the question to whether the blood feeding Triatominae subfamily is indeed a natural group (monophyletic) or not has been incidentally addressed in a few studies, but no study, thus far, was specifically designed to answer that question. The inclusion of a higher diversity of the species closely related to Triatominae and its tribes Alberproseniini, Bolboderini and Cavernicolini, and the use different analytical approaches (such as investigating metabolic pathways or transcriptomes [31]) will give better insights into the evolution of blood feeding.

\section{Triatominae systematics: taxonomy $v s$. phylogeny}

Taxonomy and phylogeny (the evolutionary relationships of taxa) are two sides of the same coin (systematics). Ideally, taxonomy reflects evolutionary relationships (i.e. a taxa are monophyletic), however due to great morphological differentiation within some lineages, lack of distinct morphological characters, and environmental influences on morphology, taxonomy may not reflect phylogeny. 
The two most well studied Triatominae tribes, Triatomini and Rhodniini, are understandably the most abundant ("easier to find") and also the most diverse ("more described species"). Built based on morphology, before DNA sequence data were readily available, both tribes have been shown to be monophyletic [1, 22-24], however new DNA based phylogenetic data show that genera within each group are not (Figure 3, Key Figure).

More specifically, in Triatomini, the clade containing the most diverse genus, Triatoma, includes all of the other six [16] genera [5,30]. A similar observation was made for the tribe Rhodniini, that comprises only two genera, Rhodnius and Psammolestes, where the latter is included in the Rhodnius lineage $[5,32,34]$. These observations make both Triatoma and Rhodnius paraphyletic. In the case of Rhodniini, however, even though Rhodnius is paraphyletic, there is evidence that Psammolestes is monophyletic $[1,26]$.

\section{Tritomini: the most diverse tribe}

The Triatomini includes 109 taxa (including the 2 fossil species), of which 84 belong to the genus Triatoma ( 1 fossil) and 15 to the genus Panstrongylus ( 1 fossil). The five other genera are: Dipetalogaster (1 species), Eratyrus (2 species), Hermanlentia (1 species), Linshcosteus (6 species) and Paratriatoma (1 species).

The genus Triatoma is not only the most diverse within the subfamily, but also the only one to occur in both the New and the Old World. High diversity can be observed not only in the number of taxa, but also in the morphological variation observed within genera. At the other end of the spectrum, molecular systematics studies have also shown several 
Triatoma taxa to be, actually, cryptic species complexes (e.g. T. sordida [36], $T$.

brasiliensis [37], T. dimidiata [38-41]. The relationship of this diversity to divergence time is addressed below.

In order to better organize the Triatoma diversity to reflect phylogeny, and with lack of evidence to raise the status of several species to genus, Usinger and collaborators [42] were the first to formally assign species to sub generic complexes (Table 1). Later on, the closely related genera Meccus, Mepraia and Nesotriatoma were synonymized to Triatoma, under the complexes phyllosoma, spinolai and flavida respectively. Currently, with the rapid collection of DNA data, there is frequent revision and no agreement amongst the numerous studies on the status of these groups. Dorn et al., recently proposed a hypothesis testing framework for Triatominae, that has yet to be applied to these data [41]. We here, suggest they all be grouped into Triatoma for the following reasons: (1) Meccus, as a genus, has been shown not to be monophyletic [30,33,43], (2) there is enough morphological and phylogenetic knowledge to include T. eratyrusiformis and T. breyeri in a monophyletic Mepraia genus [5,16,30,33], this, however has not yet been formally proposed; (3) there is not enough knowledge of the Nesotriatoma assigned species to make an evidence-based decision, and consider them a separate genus.

Interestingly enough, there has been no controversy on the change of status for Triatoma matsunoi to the monotypic genus Hermanlentia. The authors describe the characters that distinguished the species as enough to justify the description of a new genus [44] . Currently, however, in light of the deeper knowledge on Triatominae evolution, we agree that the species should be regarded as part of the Triatoma genus, as first described. 
It is true that Triatoma and Panstrongylus are, also, not monophyletic. However, for the most part, these genera have never been the subject of taxonomic dispute. So, in the interest of being consistent in the study of the Triatominae systematics, until sufficient data are available including both wide sampling and high numbers of independent genetic loci, synonymizing Mepraia, Meccus and Nesotriatoma with Triatoma, seems to be the conservative decision.

Indeed, the easiness to separate Panstrongylus from the other Triatominae, using morphology alone, is quite an interesting issue in Triatominae systematics. Despite the undisputable data showing that this is genus is not monophyletic $[5,30,33,45]$, the shape of the head ("very short and wide, with antenniferous tubercles inserted extremely close to anterior border of eyes" [6]) is consistent across the Panstrongylus genus. The head being one of the most relevant body parts in Triatominae systematics. It has been suggested that the differences observed in between the heads off adult Panstrongylus and Triatoma may be due to habitat occupation $[16,46]$, however a deeper ecological and phenotypic study on the matter is still due.

\section{Rhodniini: genetic diversity $v s$. morphological similarity}

The Rhodniini tribe comprises two genera: Rhodnius, with 20 species, and Psammolestes with 3 species (Table 1). Rhodnius is usually associated with palm trees and Psammolestes with bird nests (reviewed in [4]), which could be an explanation for the morphological discrepancy observed between the two genera. 
The genus Rhodnius is divided into three groups: the prolixus and pictipes groups distributed east of the Andes (also called cis-Andean groups), and the pallescens group west of the Andes (trans-Andean; Table 1[16,47]).

Contrasting with the morphological diversity observed in Triatoma, species assigned to the Rhodnius exhibit low morphological variation, usually being hard to identify based on morphology alone [52,53]. With further phylogenetic investigation, however, it is clear that the lack of morphological variation does not reflect low genetic diversity within the genus. Taxa that can be considered cryptic species complex, harboring several lineages under one name seem relatively more common for Rhodnius than for Triatoma [e.g. $38,39]$.

In fact, the overall morphological difference between Rhodnius and Psammolestes led to the separation of the latter into a new tribe (Psammolestiini [56]), nevertheless, when taking a closer look into characters with taxonomic relevance, Lent and Wygodzinky [6] synonymized this tribe with Rhodniini, mainly based on head morphology. The morphological characters used, however, were not enough to accurately reconstruct a cladogram and investigate the relationship between the genera.

As the relationship between the genera becomes better understood evidence suggests that, not only is Psammolestes a lineage within Rhodniini, but it seems to be part of the Rhodnius genus [32,34]. The inclusion of Psammolestes lineages in the genus Rhodnius has already been proposed [33], however, it has not been formalized yet.

The relationship among Rhodnius species groups has also been subject of disagreement over the years, mainly concerning the phylogenetic position of the pictipes group. Initially, genetic data $[32,35]$ and morphological similarities placed this as sister taxa to 
the pallescens group. Later more diverse phylogenetic and biogeographic analyses placed both cis-Andean groups as sister taxa $[5,30,33]$. This is a dispute that is still unresolved, and will benefit from the use of multi-approach evolutionary, ecological and biogeographic studies.

As in Triatoma, in Rhodnius there is an obvious tendency to focus on more epidemiologically important species. However, $R$. prolixus has also been studied because it is a model system for Heteroptera physiology and biochemistry. Combined with it being one of the most efficient $T$. cruzi vectors, this species is a very good example of how studying the evolution and ecology of cryptic vector species can be useful for vector control initiatives. Molecular phylogenetic studies revealed that $R$. robustus, morphologically indistinguishable from $R$. prolixus, is an assemblage of at least 5 lineages, all of them genetically distinct from $R$. prolixus and exclusively sylvatic $[48,49]$. With this knowledge it was possible to develop molecular tools to differentiate the real epidemiological threat, $R$. prolixus, from their look-alike counterparts, $R$. robustus [50,51].

\section{Diversification hypotheses and divergence time}

As molecular phylogenetic techniques develop and the fossil records are better known, methods have become available to date branch points in phylogenetic reconstructions. To date there are few hypotheses concerning the age of Triatominae, or how the subfamily diversified and spread. 
The first study published on ages of diversification for Triatominae lineages [58], estimated the subfamily to be as old as 100 millions of years (MY). The authors, however, included representatives of other Insect orders (i.e. Blattaria, Orthoptera, Diptera and Lepodotera), but did not include any other lineage of Hemiptera. Thus, interpretation of the age of this branch actually reflects the probable age of the order Hemiptera, and not of the subfamily Triatominae.

The use of this estimate as prior in a subsequent Triatominae dated phylogeny, supported the hypothesis that the diversification of the subfamily occurred with the formation of South America [59], a hypothesis that is arguably consistent with the geographic distribution of the group, since most of the known diversity is distributed throughout the Americas. These results, however, were obtained with the use of an age estimation extremely conservative for the Triatominae, placing the diversification too early in the Insecta tree of life.

The investigation of the divergence time of assassin bugs (Reduviidae) [29], a group that includes Triatominae, took us a step closer to understanding how and when Triatominae came to be. The broad extant and fossil Reduviidae sampling suggested the age the Triatominae as being around 35MY. It also added to the discussion concerning the monophyly of the subfamily, as in this phylogeny it was recovered as paraphyletic. Upon combining the most diverse Reduviidae sampling [29] with the most diverse on Triatominae sampling [30] it was possible to: improve accuracy on the phylogenetic reconstruction of Triatominae and estimate of divergence times for the lineages within the subfamily, and test hypotheses on vicariant events that could have led to the observed diversity of Triatominae. 
Amongst the several hypotheses tested, three of them will be highlighted here based on either previous discussion in the literature or their significance in the cladogenetic events: (1) the Rhodnius separation in cis- and trans-Andean groups, (2) the arrival of Triatominae in the Old World and (3) the rapid diversification of the Southern American Triatoma.

The classification of Rhodnius into two cis-Andean groups (pictipes and prolixus) and one tran-Andean group (pallescens), reflects high morphological similarities between the pictipes and pallescens groups. This led some authors to gather them in a single pictipes group [60] and to hypothesize that they were separated from their sibling lineages separated by the Andean uplift. Broad phylogenetic analyses show, however, the pictipes group to be sibling to prolixus, and not pallescens $[5,30,33]$. And, although the hypothesis of the Andean uplift as a vicariant event could not be completely discarded, hypothesis testing more strongly supports that the Trans-Andean group emerged as a result of dispersion [5]. It has bee suggested that Triatominae dispersed and diverged to and throughout the Old World "hitchhiking" on sailing ships as little as 300 years ago $[16,61,62]$. The age estimation of the clade, however, puts the old world dispersal event much earlier ( 20 - 25 MYA [5]), in a time where the Behring land bridge connected the Americas to Asia. Statistical analysis showed that dispersion through that bridge and the subsequent vicariant event being the disappearance of such land, could not be rejected as a viable explanation for the presence of Triatominae in the old World.

Overall, there is stronger support for the hypothesis that the Andean uplift and most of the Southern American Triatoma diversification occurred concurrently. The several climatic and ecological changes occurring in South America as a consequence of the 
uplift [63-65] led to the rapid and vast diversification of the South America Triatoma clade [5].

\section{Concluding remarks}

Genetic diversity studies in Triatominae lag behind those of other arthropod disease vectors, and, in our opinion, several factors contribute to this. Despite being one of the ten most prevalent neglected tropical diseases, Chagas disease is also a silent disease, mostly affecting neglected people. As such, in the most affected countries, resources devoted to the study of the arthropod vector of American trypanosomiasis are scarce, as are resources from wealthier, less affected countries. With such limited resources, studies are inclined to address more practical questions than to discovery driven science, and to use what is known to work instead of exploring the unknown. Table S1 shows that most phylogenetic studies use "tried and proved" markers and targeted small groups composed of mostly epidemiologically important species.

The lack of genome data, coupled with the limited sampling, resulted in a plateau in our understanding of Triatominae diversity. We believe that with the lowering costs of genome wide, high throughput sequencing methods, Triatominae systematics is now able to generate genome wide data that will be used to understand not only how the Triatominae diversified, but how this diversity relates to the unique Chagas disease transmission cycles (see Outstanding questions).

\section{Acknowledgments}


We thank Lori Stevens for reviewing our manuscript and for the valued suggestions and the anonymous reviewers for their valuable contributions towards improving this manuscript. SAJ is a Post-Doctoral Fellow funded by NSF grant BCS-1216193 as part of the joint NSF-NIH-USDA Ecology and Evolution of Infectious Diseases program and NIH grant R03AI26268/1-2. Any opinions, findings, and conclusions or recommendations expressed in this material are those of the author(s) and do not necessarily reflect the views of the National Science Foundation and of the National Institutes of Health. The funders had no role in study design.

\section{References}

1 Hotez, P.J. et al. (2008) The neglected tropical diseases of Latin America and the Caribbean: a review of disease burden and distribution and a roadmap for control and elimination. PLoS Negl. Trop. Dis. 2, e300

2 Galvão, C. et al. (2003) A Checklist of the current valid species of the subfamily Triatominae Jeannel, 1919 (Hemiptera: Reduviidae) and their geographical distribution, with nomenclatural and taxonomic notes. Zootaxa 202, 1-36

3 Brenière, S.F. et al. (2016) Over Six Thousand Trypanosoma cruzi Strains Classified into Discrete Typing Units (DTUs): Attempt at an Inventory. PLoS Negl. Trop. Dis. 10, e0004792

4 Galvao, C. and Justi, S.A. (2015) An overview on the ecology of Triatominae (Hemiptera:Reduviidae) Cleber. Acta Trop.

5 Justi, S.A. et al. (2016) Geological Changes of the Americas and their Influence on the Diversification of the Neotropical Kissing Bugs (Hemiptera: Reduviidae: Triatominae). PLoS Negl. Trop. Dis. 10, e0004527

6 Lent, H. and Wygodzinsky, P. (1979) Revision of the Triatominae (Hemiptera: reduviidae), and their significance as vectors of Chagas Disease. Bull. Am. Museum Nat. Hist. 163, 123-520

7 García, B.A. and Powell, J.R. (1998) Phylogeny of Species of Triatoma (Hemiptera: Reduviidae) Based on Mitochondrial DNA Sequences. J. Med. Entomol. 35, 232-238

8 Dotson, E.M. and Beard, C.B. (2001) Sequence and organization of the mitochondrial genome of the Chagas disease vector, Triatoma dimidiata. Insect Mol. Biol. 10, 205-215

9 Logue, K. et al. (2013) Mitochondrial genome sequences reveal deep divergences 
among Anopheles punctulatus sibling species in Papua New Guinea. Malar. J. 12, 64

10 Neafsey, D.E. et al. (2015) Highly evolvable malaria vectors: The genomes of 16 Anopheles mosquitoes. Science (80-. ). DOI: 10.1126/science.1258522

11 Mesquita, R.D. et al. (2016) Genome of Rhodnius prolixus, an insect vector of Chagas disease, reveals unique adaptations to hematophagy and parasite infection. Proc. Natl. Acad. Sci. 113, 201600205

12 Gourbière, S. et al. (2012) Genetics and evolution of triatomines: from phylogeny to vector control. Heredity (Edinb). 108, 190-202

13 Schofield, C.J. (1988) The biosystematics of Triatominae. In Biosystematics of Haematophagous Insects. Systematics Association special volume 37 pp. 284-312

14 Schaefer, C.W. (2003) Triatominae (Hemiptera: Reduviidae): systematic questions and some others. Neotrop. Entomol. 32, 1-10

15 Schaefer, C.W. (2005) Why are the subfamily relationships of Triatominae (Hemiptera: Reduviidae) important? Entomol. y Vectores 12, 19-35

16 Schofield, C.J. and Galvão, C. (2009) Classification, evolution, and species groups within the Triatominae. Acta Trop. 110, 88-100

17 Sandoval, C. et al. (2010) Feeding behaviour of Belminus ferroae (Hemiptera: Reduviidae), a predaceous Triatominae colonizing rural houses in Norte de Santander, Colombia. 2Medical Vet. Entomol. 24, 124-131

18 Sandoval, C. et al. (2004) Feeding sources and natural in- fection of Belminus herreri (Hemiptera, Reduviidae, Triatominae) from dwellings in Cesar, Colombia. Mem. Inst. Oswaldo Cruz 99, 137-140

19 Calegari, L. et al. (1995) Habitos alimentarios de Triatoma rubrovaria (Blanchard, 1843) (Hemiptera, Triatominae), en diferentes situaciones eco-epidemiologicas. Bol Soc Zool Uruguay 9, 61-66

20 Mendonça, V.J. et al. (2016) Revalidation of Triatoma bahiensis Sherlock \& Serafim, 1967 (Hemiptera: Reduviidae) and phylogeny of the T. brasiliensis species complex. Zootaxa 4107, 239-254

21 Freitas, S.P.C. et al. (2005) Fontes alimentares de Triatoma pseudomaculata no Estado do Ceará , Brasil Feeding patterns of Triatoma pseudomaculata in the state of Ceará, Brazil. Rev. Saúde Pública 39, 27-32

22 Durán, P. et al. (2016) On triatomines, cockroaches and haemolymphagy under laboratory conditions : new discoveries. 111, 605-613

23 de Paula, A.S. et al. (2005) Testing the sister-group relationship of the Rhodniini and Triatomini (Insecta: Hemiptera: Reduviidae: Triatominae). Mol. Phylogenet. Evol. 35, 712-8

24 Weirauch, C. (2008) Cladistic analysis of Reduviidae (Heteroptera: Cimicomorpha) based on morphological characters. Syst. Entomol. 33, 229-274

25 Weirauch, C. and Munro, J.B. (2009) Molecular phylogeny of the assassin bugs 
(Hemiptera: Reduviidae), based on mitochondrial and nuclear ribosomal genes. Mol. Phylogenet. Evol. 53, 287-99

26 Heath, T.A. et al. (2008) Taxon sampling and the accuracy of phylogenetic analyses. J. os Sytematics Evol. 46, 239-257

27 Graybeal, A. (1998) Is It Better to Add Taxa or Characters to a Difficult Phylogenetic Problem? Syst. Biol. 47, 9-17

28 Takahata, N. and Nei, M. (1985) Gene genealogy and variance of interpopulational nucleotide differences. Genetics 110, 325-344

29 Hwang, W.S. and Weirauch, C. (2012) Evolutionary history of assassin bugs (insecta: hemiptera: Reduviidae): insights from divergence dating and ancestral state reconstruction. PLoS One 7, e45523

30 Justi, S.A. et al. (2014) Molecular phylogeny of Triatomini (Hemiptera: Reduviidae: Triatominae). Parasit. Vectors 7, 149

31 Zhang, J. et al. (2016) Evolution of the assassin's arms: insights from a phylogeny of combined transcriptomic and ribosomal DNA data (Heteroptera: Reduvioidea). Sci. Rep. 6, 22177

32 Lyman, D.F. et al. (1999) Mitochondrial DNA sequence variation among triatomine vectors of Chagas' disease. Am. J. Trop. Med. Hyg. 60, 377-86

33 Hypsa, V. et al. (2002) Phylogeny and biogeography of Triatominae (Hemiptera: Reduviidae): molecular evidence of a New World origin of the Asiatic clade. Mol. Phylogenet. Evol. 23, 447-57

34 Monteiro, F. et al. (2002) Allozyme relationships among ten species of Rhodniini, showing paraphyly of Rhodnius including Psammolestes. Med. Vet. Entomol. 16, 83-90

35 Monteiro, F. a et al. (2000) Phylogeny and molecular taxonomy of the Rhodniini derived from mitochondrial and nuclear DNA sequences. Am. J. Trop. Med. Hyg. $62,460-5$

36 Noireau, F. et al. (1998) Cryptic speciation in Triatoma sordida (Hemiptera:Reduviidae) from the Bolivian Chaco. Trop. Med. Int. Health 3, 36472

37 Monteiro, F.A. et al. (2004) Nested clade and phylogeographic analyses of the Chagas disease vector Triatoma brasiliensis in Northeast Brazil. Mol. Phylogenet. Evol. 32, 46-56

38 Bargues, M.D. et al. (2008) Phylogeography and genetic variation of Triatoma dimidiata, the main Chagas disease vector in Central America, and its position within the genus Triatoma. PLoS Negl. Trop. Dis. 2, e233

39 Dorn, P.L. et al. (2009) Two distinct Triatoma dimidiata (Latreille, 1811) taxa are found in sympatry in Guatemala and Mexico. PLoS Negl. Trop. Dis. 3, e393

40 Monteiro, F. a. et al. (2013) Phylogeographic Pattern and Extensive Mitochondrial DNA Divergence Disclose a Species Complex within the Chagas Disease Vector 
Triatoma dimidiata. PLoS Negl. Trop. Dis. 8, e70974

41 Dorn, P.L. et al. (2016) Hypothesis testing clarifies the systematics of the main Central American Chagas disease vector, Triatoma dimidiata (Latreille, 1811), across its geographic range. Infect. Genet. Evol. 44, 431-443

42 Usinger, R.L. et al. (1966) The biosystematics of Triatominae. Annu. Rev. Entomol. 11, 309-30

43 Ibarra-Cerdeña, C.N. et al. (2014) Phylogeny and Niche Conservatism in North and Central American Triatomine Bugs (Hemiptera: Reduviidae: Triatominae), Vectors of Chagas' Disease. PLoS Negl. Trop. Dis. 8, e3266

44 Jurberg, J. and Galvao, C. (1997) Hermanlentia n.gen. da tribo Triatomini, com um rol de espécies de Triatominae (Hemiptera, Reduviidae). Mem. Inst. Oswaldo Cruz 92, 181-185

45 Marcilla, a et al. (2002) Nuclear rDNA ITS-2 sequences reveal polyphyly of Panstrongylus species (Hemiptera: Reduviidae: Triatominae), vectors of Trypanosoma cruzi. Infect. Genet. Evol. 1, 225-35

46 Patterson, J.S. et al. (2008) On the genus Panstrongylus Berg 1879: Evolution, ecology and epidemiological significance. Acta Trop. DOI: 10.1016/j.actatropica.2008.09.008

47 Abad-franch, F. et al. (2009) Ecology, evolution, and the long-term surveillance of vector-borne Chagas disease : A multi-scale appraisal of the tribe Rhodniini (Triatominae). Acta Trop. 110, 159-177

48 Monteiro, F. a et al. (2003) Molecular phylogeography of the Amazonian Chagas disease vectors Rhodnius prolixus and R. robustus. Mol. Ecol. 12, 997-1006

49 Pavan, M.G. et al. (2015) Looks Can be Deceiving: Cryptic Species and Phenotypic Variation in Rhodnius spp., Chagas Disease Vectors. In Evolutionary Biology: Biodiversification from Genotype to Phenotype pp. 345-372

50 Pavan, M.G. and Monteiro, F. a (2007) A multiplex PCR assay that separates Rhodnius prolixus from members of the Rhodnius robustus cryptic species complex (Hemiptera: Reduviidae). Trop. Med. Int. Health 12, 751-8

51 Pavan, M.G. et al. (2013) A nuclear single-nucleotide polymorphism (SNP) potentially useful for the separation of Rhodnius prolixus from members of the Rhodnius robustus cryptic species complex (Hemiptera: Reduviidae). Infect. Genet. Evol. 14, 426-33

52 Lent, H. (1948) O genero Rhodnius Stål, 1859 (Hemiptera, Reduvioidea). Rev. Bras. Biol. 8, 297-339

53 Neiva, A. and Pinto, C. (1923) Estado actual dos conhecimentos sôbre o gênero Rhodnius Stål, com a descripção de uma nova especie. Bras. Médico 37, 20-24

54 Diaz, S. et al. (2014) Genetic, cytogenetic and morphological trends in the evolution of the Rhodnius (Triatominae: Rhodniini) trans-Andean group. PLoS One 9, 1-12 
55 Monteiro, F. a et al. (2003) Molecular phylogeography of the Amazonian Chagas disease vectors Rhodnius prolixus and R. robustus. Mol. Ecol. 12, 997-1006

56 Carcavallo, R.U. (1976) Aspects of the epidemiology of Chagas disease in Venezuela and Argentina. PAHO Sci. Publ. 318, 347-358

57 Wiens, J.J. and Tiu, J. (2012) Highly Incomplete Taxa Can Rescue Phylogenetic Analyses from the Negative Impacts of Limited Taxon Sampling. 7,

58 Gaunt, M.W. and Miles, M. a (2002) An insect molecular clock dates the origin of the insects and accords with palaeontological and biogeographic landmarks. Mol. Biol. Evol. 19, 748-61

59 Patterson, J.S. and Gaunt, M.W. (2010) Phylogenetic multi-locus codon models and molecular clocks reveal the monophyly of haematophagous reduviid bugs and their evolution at the formation of South America. Mol. Phylogenet. Evol. 56, 60821

60 Abad-Franch, F. and Monteiro, F. a (2007) Biogeography and evolution of Amazonian triatomines (Heteroptera: Reduviidae): implications for Chagas disease surveillance in humid forest ecoregions. Mem. Inst. Oswaldo Cruz 102 Suppl, 5770

61 Gorla, D.E. et al. (1997) Biosystematics of Old World Triatominae. Acta Trop. 63, $127-40$

62 Patterson, J.S. et al. (2001) Population morphometric analysis of the tropicopolitan bug Triatoma rubrofasciata and relationships with old world species of Triatoma: evidence of New World ancestry. Med. Vet. Entomol. 15, 443-51

63 Vries-laboratorium, C.H.H. De et al. (1995) Andean tectonics as a cause for changing drainage patterns in Miocene northern South America.

64 Hoorn, C. et al. (2010) Amazonia Through Time : Andean. Science (80-. ). 330, 927-931

65 Batalha-Filho, H. et al. (2013) Connections between the Atlantic and the Amazonian forest avifaunas represent distinct historical events. J. Ornithol. 154, $41-50$

gene suggests passive dispersal of the Panstrongylus megistus population in Brazil. 39, $441-444$

\section{Figure legends}

Figure 1. Types of homology. Homology is similarity by descent. When a gene duplication occurs, it generates two distinct types of homology: orthology and paralogy. Assume a gene duplication took place, generating copies A and B. All descendants from 
copy A will be orthologs among themselves. Same for copy B descendants. However, copy A descendants will be paralogs regarding copy B descendants, and vice-versa. Yet, they are all still homologs, because they all descend from the gene that duplicated in the first place.

Figure 2. Types of taxonomic groups. I) Groups Red and Blue are both monophyletic, because they include all the descendants of a most recent common ancestor (MRCA) and the ancestor. II) Group Blue is polyphyletic, because it includes lineages that evolved independently. III) Groups Red and Blue are paraphyletic because they do not include all the descendants from the MRCA.

Figure 3, Key Figure. Consensus phylogenetic relationships amongst members of the Triatomini and Rhodniini tribes based on published phylogenies. Photos of the specimens represent the type species for all the genera and are not to scale NH Triatoma, Triatoma from the Northern Hemisphere; SH Triatoma, Triatoma from the Southern Hemisphere. Images courtesy of Laboratorio Nacional e Internacional de Referencia em Taxonomia de Triatomineos, LNIRTT, Oswaldo Cruz Institute, FIOCRUZ. 
Table 1. List of Triatominae valid species and respective classification into groups and complexes.

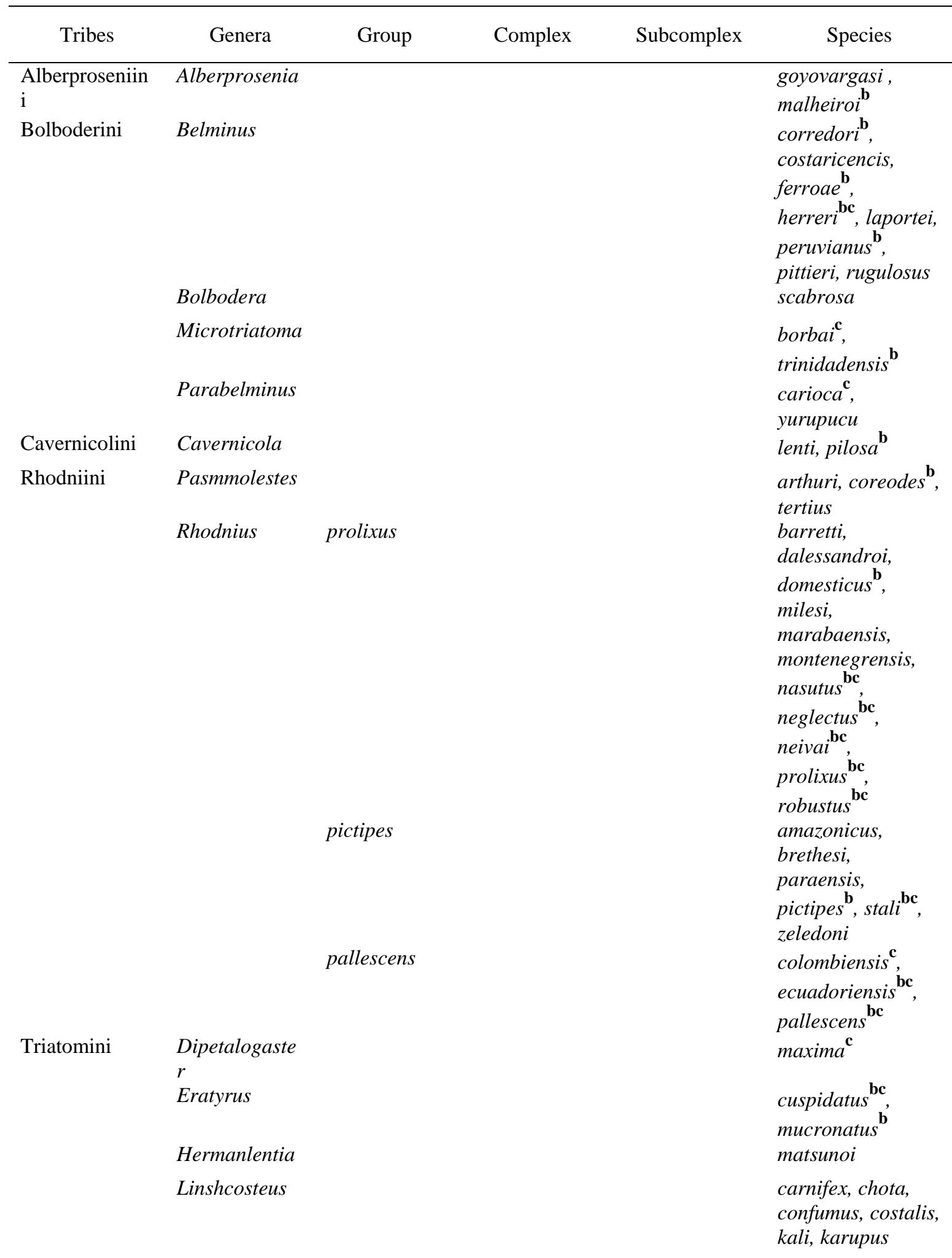


Panstrongylus

ParaTriatoma

Triatoma

rubrofasciat

a

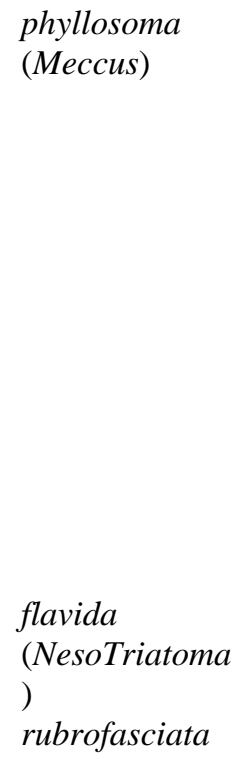

protracta

lecticularia

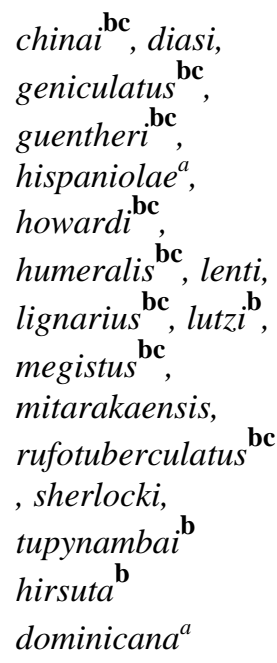




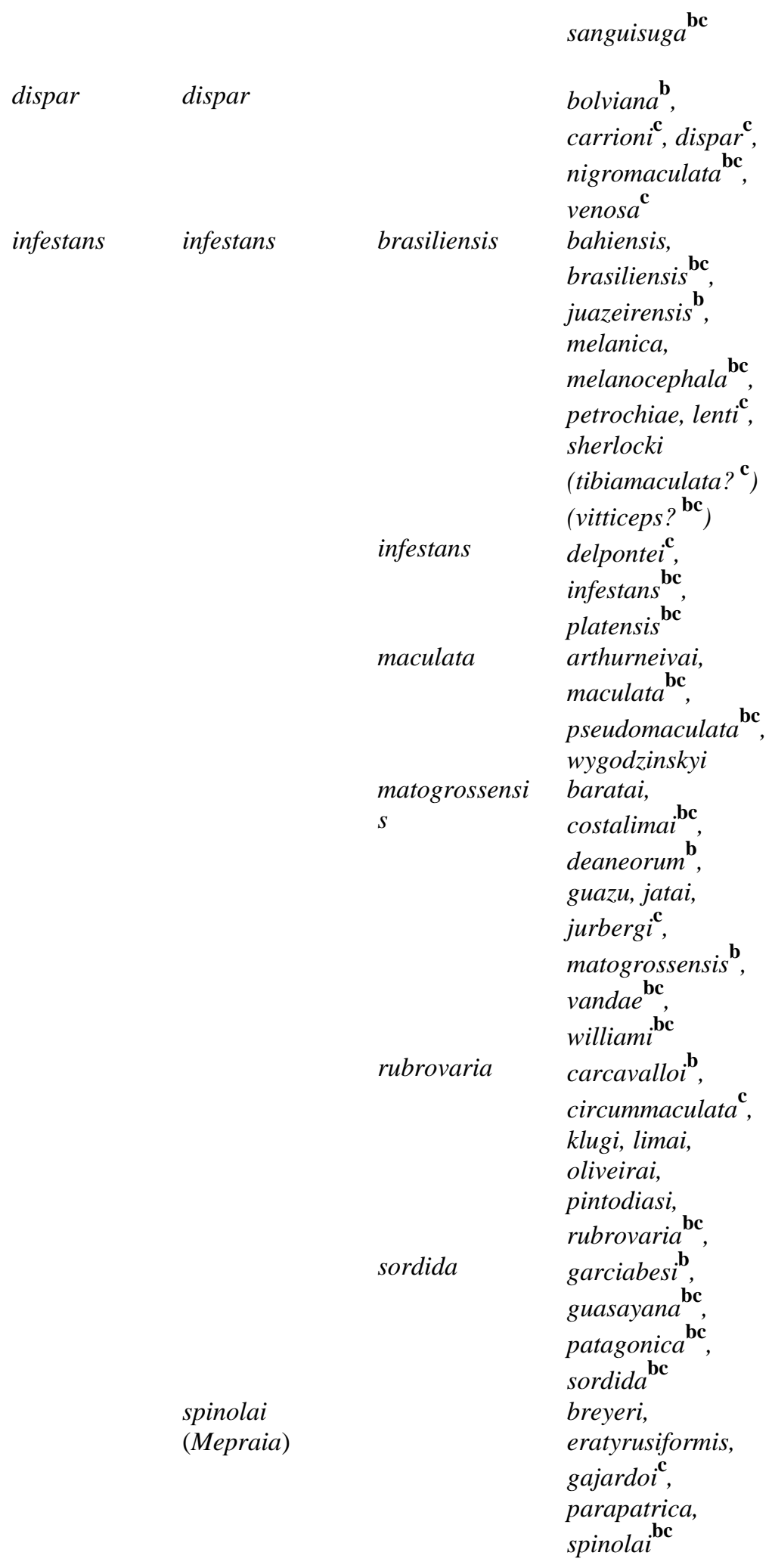

${ }^{\mathrm{a}}$ Fossil species; ${ }^{\mathrm{b}}$ species that have been found in domestic or peridomestic environment; ${ }^{\mathrm{c}}$ species that have been found infected with T. cruzi [4]. 


\section{Glossary}

-omics data: refers to data generated through whole genome, transcriptome and proteome sequencing.

Clade: representation of a group of organisms and their ancestor.

Cladistic analyses: study of relationships between organisms based on shared characters derived from the most recent common ancestor (MRCA). Historically done using morphological characters, displaying results in a graph referred to as cladogram.

Cladogenetic events: separation of one independently evolving lineage into two or more independent new lineages. When a cladogenetic event occurs, the ancestor population (independently evolving lineage) becomes the MRCA for the new formed lineges.

Cryptic species: species that are morphologically indistiguishible, more frequently recognized because of genetic differences. Biological differences may or may not be detectable.

Domestication: in Triatominae, domestication is used as the ability of the insects to form colonies inside human dwellings.

Extant: refers to lineages that are living now, as opposed to extinct ones. 
Gene trees: graphic representation of the evolutionary history of a single gene within a set of organisms.

Genome: whole content of coding and non-coding DNA from a given organism.

Hematophagous: an organism that feeds on blood.

Lineages: isolated and independently evolving group of organisms.

MRCA: most recent common ancestor.

Mitochondrial markers: identifiable portions of DNA (coding or non-coding) from the mitochondrial genome.

Molecular phylogeny: study of the relationship between organisms using molecular markers for character comparison. Results are usually displayed in a graph called phylogenetic tree, and the process of obtaining that tree (i.e. the analysis) is referred to as phylogenetic reconstruction.

Monophyletic: a clade that included the MRCA and all its descendants.

Monotypic: a taxon that has only on sub-taxon assigned to it (i.e. a monotypic genus has only one species described).

Multi-copy nuclear ribosomal markers: portions of the nuclear DNA that code for ribosomal RNA and that appear in several copies scattered throughout the genome.

Paraphyletic: a clade that does not include all the descendants of the MRCA from a group of organisms.

Polyphyletic: an assemblage that includes independently evolving lineages under the same name. 
Sister (lineages/taxon): in a cladogenetic event, the MRCA generates new independently evolving lineages that are most closely related to each other than to any other lineages. Those are called sisters lineages. If each of the lineages is given a name, those are sister taxa.

Species trees: graphic representation of the evolutionary history of a group of organisms. A species tree usually contains several distinct gene trees within it.

Taxon (pl. Taxa): a group of organisms recognized as a unit.

Taxonomy: description, identification and classification of organisms.

Transcriptome: mRNA content of a given organism or tissue.

Type species: The name-bearing type of a nominal genus or subgenus (http://iczn.org/iczn/index.jsp)

VectorBase: NIAID Bioinformatics Resource Center providing genomic, phenotypic and population-centric data to the scientific community for invertebrate vectors of human pathogens (www.vectorbase.org). 


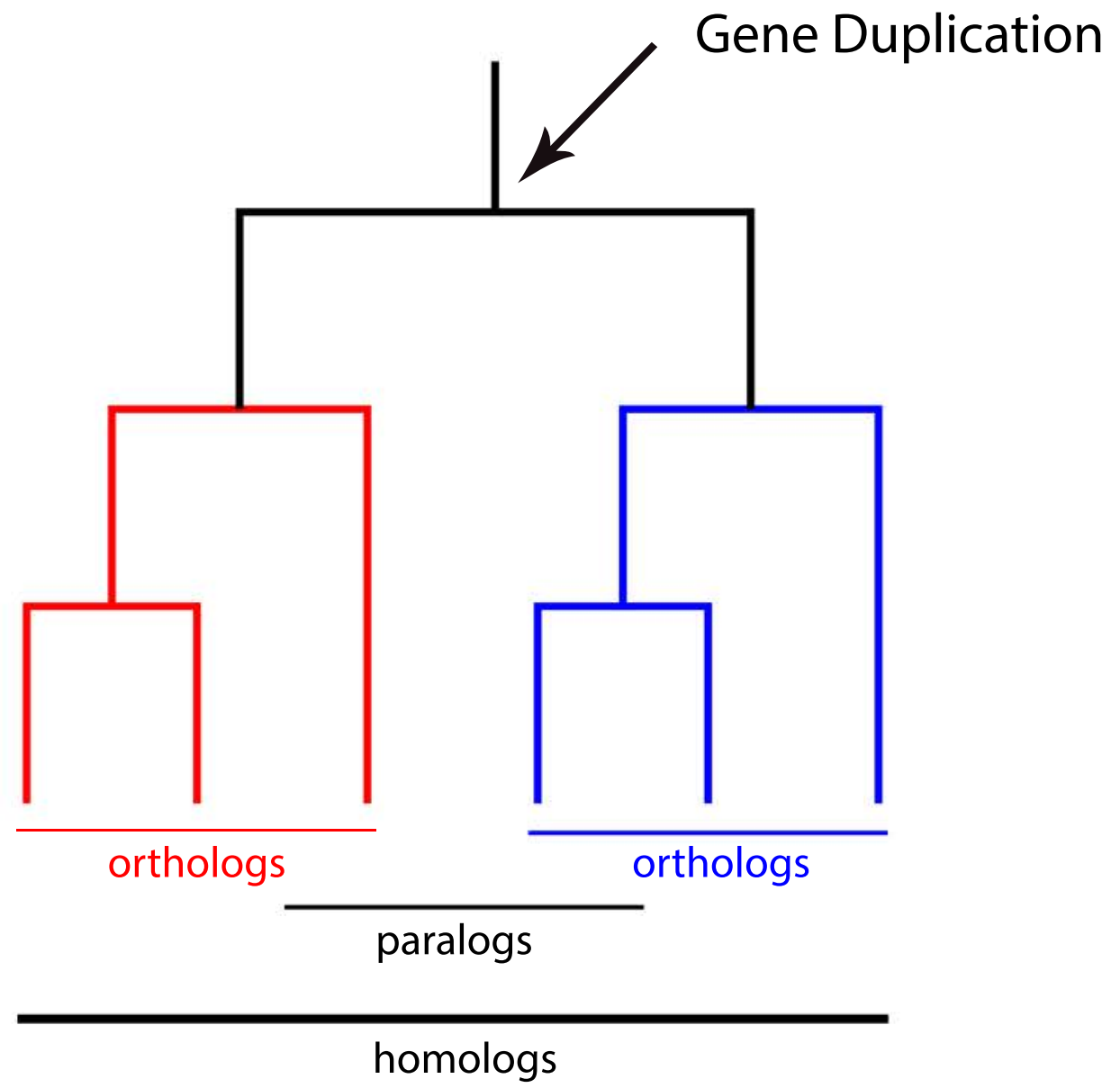


A)

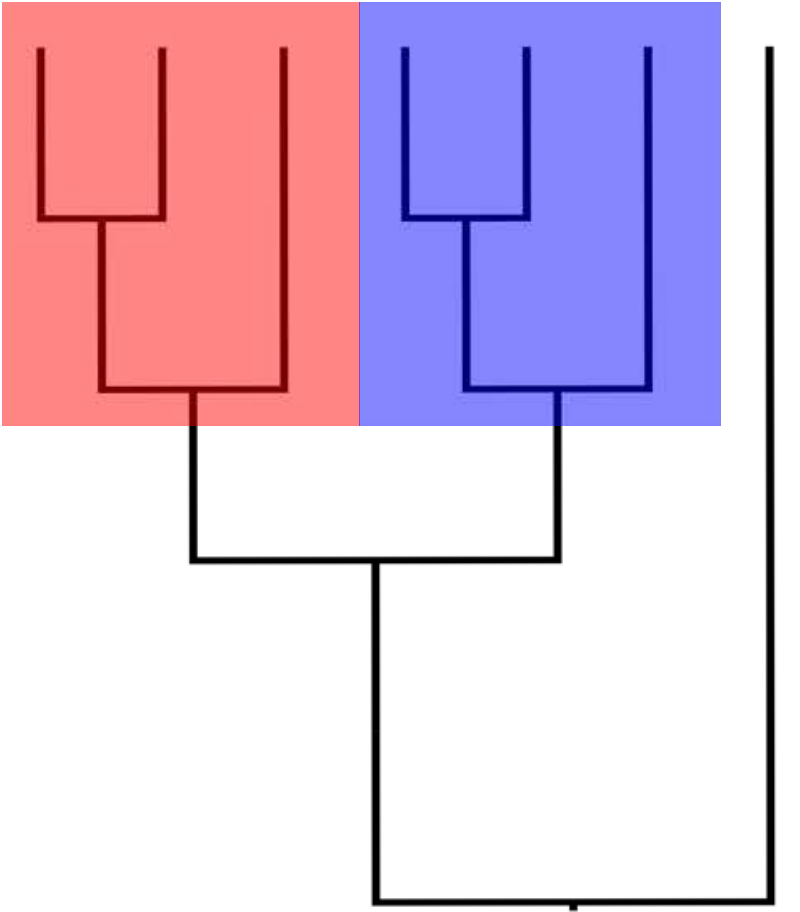

B)

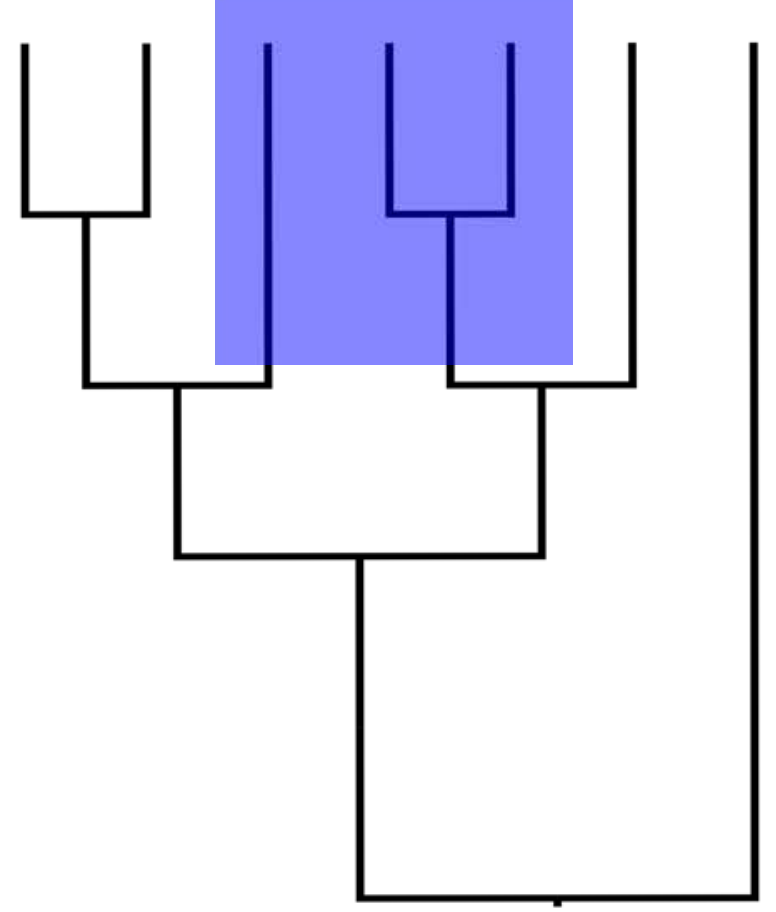

C)

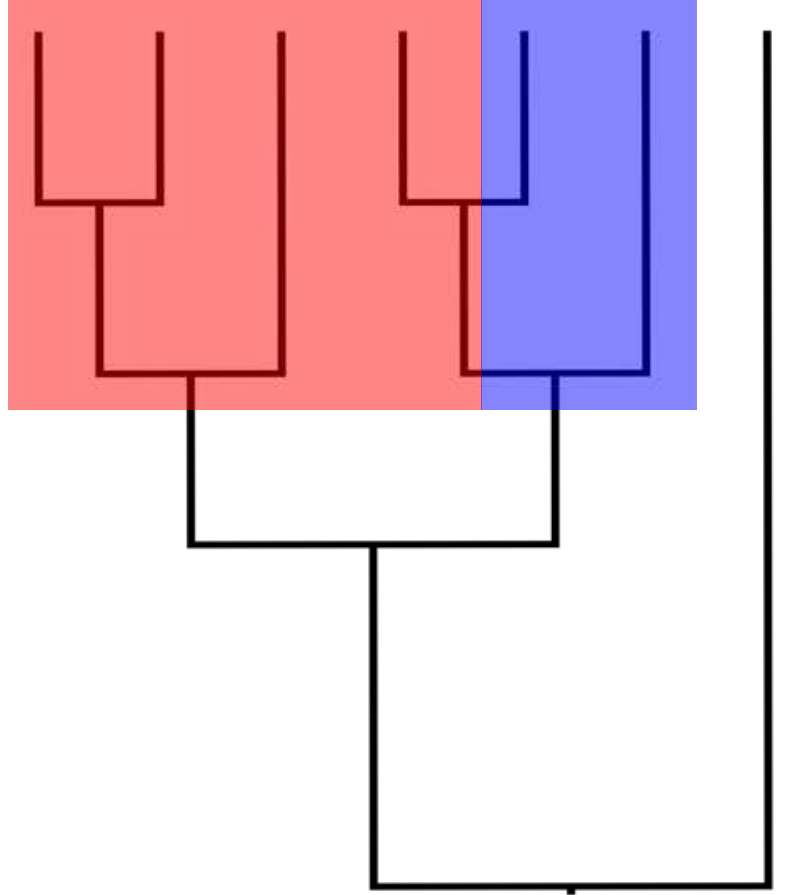


Click here to download Key Figure Figure3_Key_Figure reduced.pdf \pm
Panstrongylus

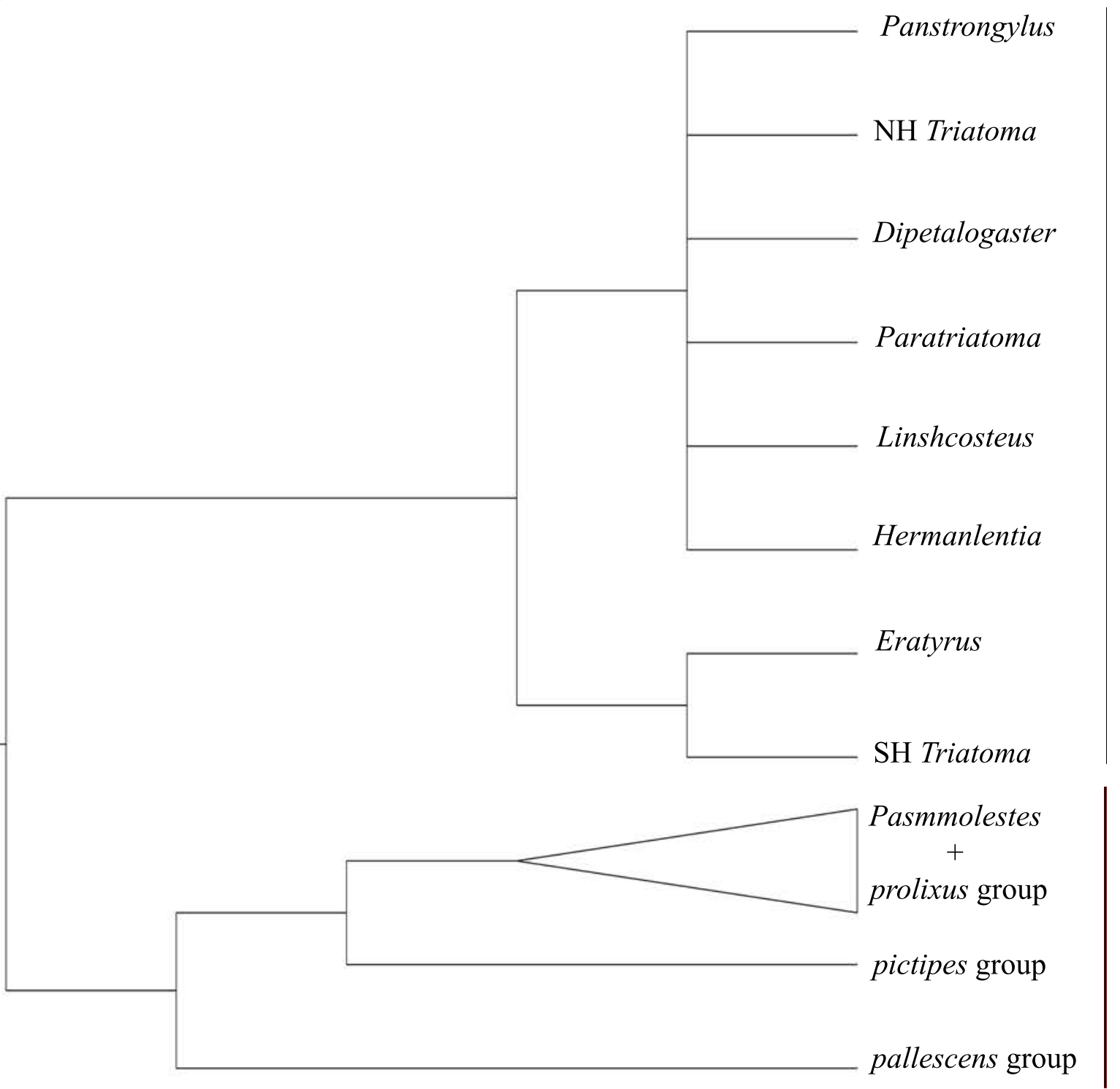

T

P. guentheri

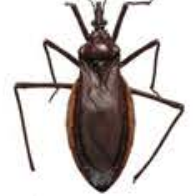

D. maxima

r

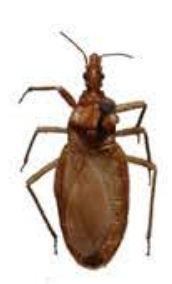

Pa.hirsuta

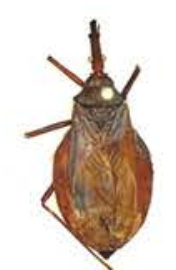

H. matsunoi

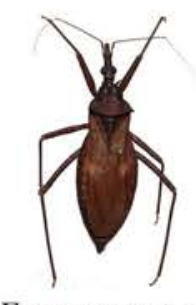

E. mucronatus

L. carnifex
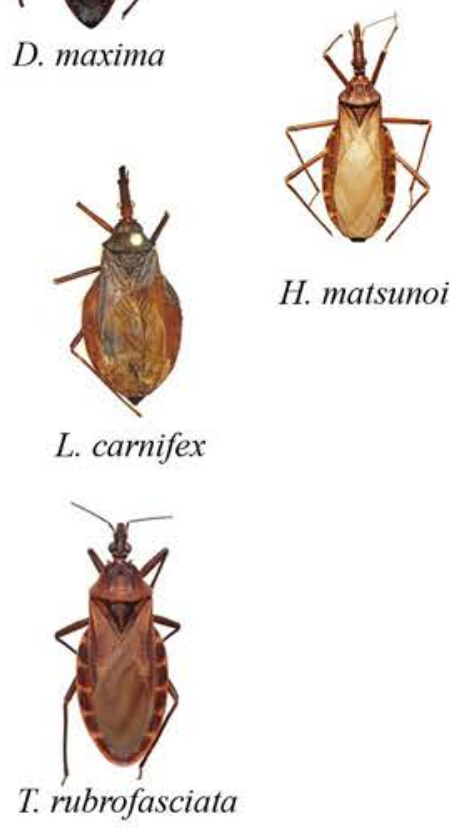

\begin{tabular}{|c|c}
$\mathrm{R}$ \\
$\mathrm{h}$ \\
$\mathrm{o}$ \\
$\mathrm{d}$ \\
$\mathrm{n}$ \\
$\mathrm{i}$ \\
$\mathrm{i}$ \\
$\mathrm{n}$ \\
$\mathrm{i}$
\end{tabular}
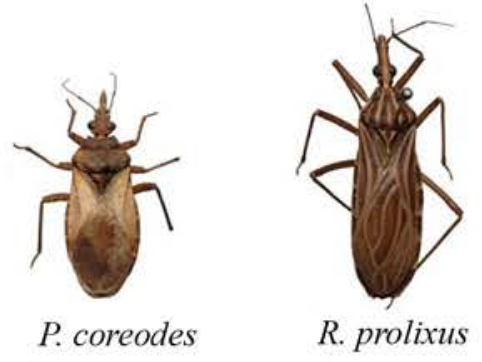

outgroup 